\section{El papiro de Edwin Smith y su trascendencia médica y odontológica}

\author{
ALEX VARGAS ${ }^{1, a}$, MARCELO LÓPEZ ${ }^{2, b}$, \\ CLAUDIO LILLO ${ }^{3, \mathrm{c}}$, MARÍA JOSEFINA VARGAS ${ }^{4, a}$
}

\section{The Edwin Smith papyrus in the history of medicine}

The historical bases of occidental medicine precede the Hippocratic corpus. Between the third and first millennium B.C. Egyptian medicine developed a model of medical practice that was a reference horizon for other Mediterranean cultures. There are a great number of papyri of that time, which gathered the medical and surgical skills and that are matter of study. The Edwin Smith papyrus (PES) is one of them. We analyzed the PES in its historical context, its history, its structure and its medical and dental significance. Finally, we analyzed the relevance of PES as a sign of a change in the medicine study method in the ancient Egypt. PES is an insight into how medicine was practiced in ancient Egypt. Historically, it is also the first medical document based on objective observations, excluding all magical and religious perceptions, as well as the underlying cultural framework. The similarity between the current clinical method and that described in the Smith papyrus, strongly suggests the idea that part of the origin of medicine, can be found in ancient Egypt.

(Rev Med Chile 2012; 140: 1357-1362).

Key words: Chronology as topic; History of Medicine, ancient.

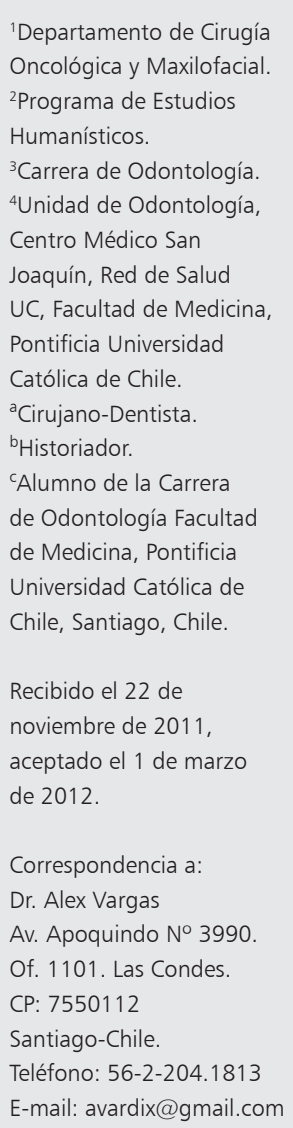

'Departamento de Cirugía

${ }^{2}$ Programa de Estudios

Humanísticos.

${ }^{3}$ Carrera de Odontología.

${ }^{4}$ Unidad de Odontología

Centro Médico San

Joaquín, Red de Salud

UC, Facultad de Medicina

Pontificia Universidad

Católica de Chile.

${ }^{a}$ Cirujano-Dentista.

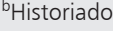

'Alumno de la Carrera

Chile, Santiago, Chile.

Recibido el 22 de

noviembre de 2011

aceptado el 1 de marzo

E-mail:avardix@gmail.com
L a historia de la práctica y saber médicos en Occidente suelen remitirnos a un origen que se circunscribe al complejo de culturas que existieron en el Mediterráneo y, en forma particular, a la medicina hipocrática. Sustentada en la concepción de la physis, la base racional de la medicina griega se convirtió en un sistema canónico que, con una serie de añadidos posteriores, gozó de cierta legitimidad hasta los albores de la sociedad industrial.

No obstante, es interesante mencionar que, desde una perspectiva histórico-médica que enfatiza el perfil racional del acto médico, las bases históricas de la medicina occidental preceden al corpus hipocrático. Específicamente, hablamos de la medicina egipcia que fue capaz de elaborar entre los milenios tercero y primero a.C. un modelo de práctica médica que en gran medida se constituyó en horizonte de referencia para otras culturas mediterráneas (Creta, Grecia), de África (Etiopía y Libia) y Asia central.

Ahora, es parte del debate histórico determinar los niveles de racionalismo que nutrieron la medicina que se desarrolló en el Nilo de los faraones. Algunos historiadores, como Roy Porter, sostienen que el conjunto de conocimientos médicos egipcio fue un híbrido que reunió componentes empíricos junto a creencias mágico-religiosas ${ }^{1}$. En la misma línea, Paul Ghalioungui, afirma que desde una perspectiva general la medicina egipcia, y conforme a las fuentes que se manejan, muchas de ellas "débiles e inciertas", reviste aspecto mágico, pero, a la vez, cuenta con "un fondo válido

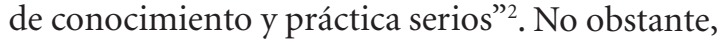
otros investigadores son más enfáticos al sostener que el antiguo Egipto fue la cuna del "pensamiento analítico médico" y que ahí se hallan las raíces médicas de Occidente, o al menos su base práctica ${ }^{3}$. 
Con todo, la serie de papiros, que reunió a modo de enciclopedia los saberes y prácticas en torno a diagnóstico, técnicas quirúrgicas y farmacopea; constituye una fuente para conocer históricamente una fase de la gestación de la medicina occidental y su racionalismo científico. Y, precisamente, una de las fuentes que permite sostener la visión racionalista es el papiro de Smith.

\section{Historia del papiro}

El papiro lleva el nombre de Edwin Smith (1822-1906) (Figura 1), que nació en Connecticut y se destacó por ser un aventurero traficante de antigüedades. Tempranamente se interesó en la egiptología, estudiando su idioma tanto en Londres como en París. A principios de 1858 se trasladó a Luxor, ciudad egipcia edificada sobre las ruinas de Tebas, que fuera la capital del Imperio Nuevo del antiguo Egipto en el siglo XVI a.C ${ }^{4}$.

Fue en esta ciudad, en 1862, donde Smith adquirió el papiro de las manos de Mustafá Aga, un negociante nativo, quien malintencionadamente lo había partido en dos partes. Smith se dio el trabajo de juntar ambos trozos guiándose por sus símbolos, elementos y estructura, e intentó infructuosamente traducirlo. Smith siempre supo de la gran importancia del papiro reconociéndolo como un tratado de medicina, sin embargo, nunca lo publicó ${ }^{5}$.

No fue hasta su muerte en 1906 que le dejó el papiro a su hija, quien lo donó a la Sociedad de Historia de Nueva York. En 1920, la traducción del papiro fue encargada a James Henry Breasted (1865-1953) (Figura 2), quien se desempeñaba en esa época como director del Instituto de Estudios Orientales de la Universidad de Chicago. Breasted se ocupó durante varios años de la traducción y edición del papiro junto al Dr. Arno B. Luckhardt, profesor de Fisiología de la misma institución y amante de la historia de la medicina, aportando sus conocimientos en los términos médicos presentes en el texto. Actualmente, el papiro se encuentra en el Museo Metropolitano de Arte de Nueva York, disponible en versión digital y con aportes de una nueva traducción encargada al egiptólogo James P. Allen ${ }^{6}$.

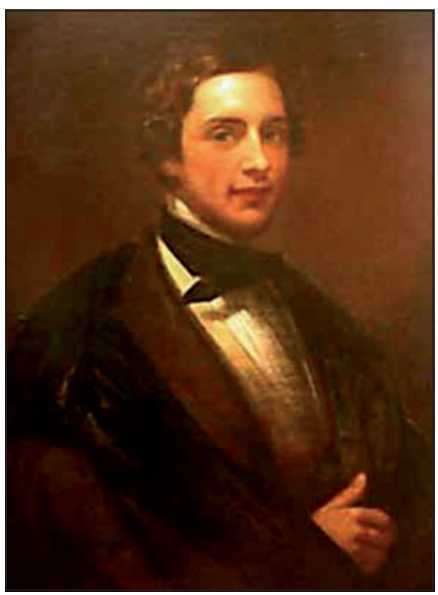

Figura 1. Edwin Smith. Pintura al óleo realizada por Francesco Anelli, de la colección de la New York Historical Society. Tomado de González Fisher R, Flores P. El Papiro Quirúrgico de Edwin Smith. An Med Asoc Med Hosp, 2005. 50 (1): p. 43-8. (5)

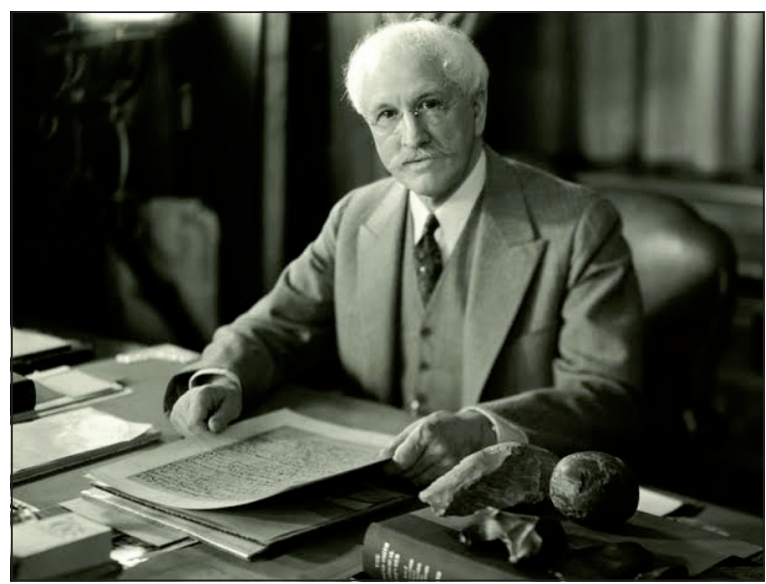

Figura 2. James Henry Breasted. Tomada del portal web Philosophy of Science Portal hhttp://philosophyofscienceportal. blogspot.com/2010/07/james-henry-breastedone-of-giants-in. html. Consultado el 21 de noviembre de 2011).

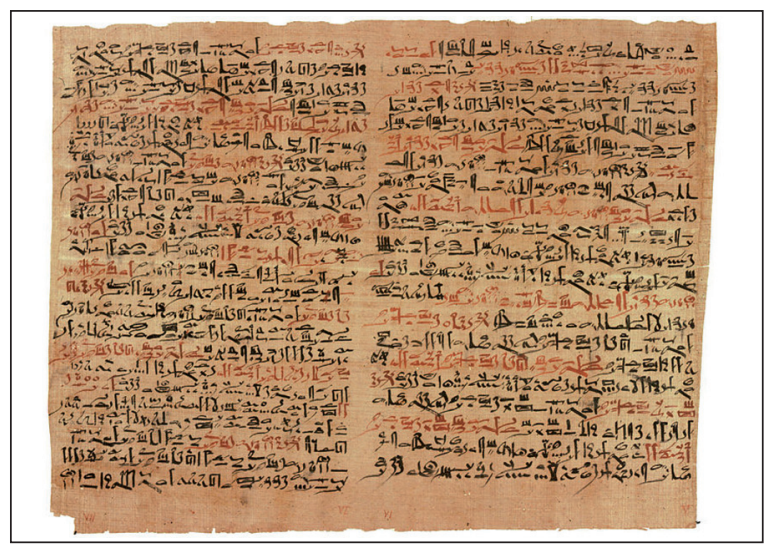

Figura 3. Extracto del Papiro de Edwin Smih. Tomado de la Versión digital en Turning the Pages. Disponible en http://archive. hlm.nih.gov. [Consultado el 13 de noviembre de 2011]. 
El Papiro de Edwin Smith (Figura 3) data del 1600 a.C. pero se cree es copia de un papiro mucho más antiguo, quizás del 3000 a.C., el cual podría corresponder al "Libro Secreto del Médico". Breasted propuso la hipótesis de que el autor del texto original podría ser el mismo Imhotep (2600 a.C.) quien fuera un gran arquitecto, médico, sacerdote, astrónomo, escriba, consejero y científico egipcio que vivió en la misma fecha en que probablemente fue escrito el papiro original. Sin embargo, el hecho de que este papiro sea de carácter objetivo y científico y carente de oraciones, conjuros y prescripciones fantasiosas, hace rechazar esta idea, ya que Imhotep era particularmente religioso.

Quien haya sido el autor del papiro original utilizó términos arcaicos propios de la época, los cuales son aclarados como nota al margen o "glosas" por otro autor, probablemente médico. La copia del texto original fue realizada en el 1600 a.C. por un autor desconocido y es el texto que se conoce como Papiro de Edwin Smith. Esta copia está inconclusa y con varios errores, lo que hace suponer que este segundo autor no era médico ${ }^{7}$. Está escrito en segunda persona singular, es decir, con el pronombre "Tú", lo que hace suponer que el papiro era un instructivo de cirugía utilizado por los cirujanos egipcios en su rutina diaria o un libro de enseñanza de los cirujanos egipcios hacia sus alumnos. Esto último debido a la gran cantidad de información que éste contiene-lo que dificulta su transmisión oral-, además de las notas y comentarios al margen, que pueden corresponder a las discusiones y debates que hayan tenido profesores con alumnos. También puede corresponder a un cuaderno de apuntes de estudiantes de medicina y cirugía.

\section{Su estructura}

El papiro (Figura 3) mide entre 32,5 y 33 centímetros de alto, medida característica de los rollos de la época de los imperios medio y antiguo; y 4,68 metros de largo. Sin embargo, se cree podría llegar a medir 5 metros debido a que una columna está perdida. Consta de 21 columnas, 17 por la parte anterior (recto) y 4,5 por la parte posterior (verso).

La escritura utilizada son rúbricas, diferenciando con tinta roja las conclusiones y con tinta negra el inicio de cada caso.

Las 17 columnas de la parte anterior corres- ponden al tratado quirúrgico propiamente tal. Consta de 48 casos ordenados topográficamente según la ubicación de la lesión en sentido céfalo caudal. En cada grupo las lesiones están ordenadas según la severidad de la lesión en orden creciente. Cada lesión está estructurada comenzando con un título, luego un examen, un diagnóstico, un tratamiento y finalmente un comentario y glosas. El Título identifica la ubicación y órganos afectados por la lesión; el Examen corresponde enteramente a semiología, es decir, inspección, palpación, auscultación y en algunos casos tomar el olor de la lesión, además de lo relatado por el paciente. Sigue el Diagnóstico que, repitiendo el enunciado del título de la lesión, decide si esa lesión será tratada, no tratada o se luchará por ella. Luego, el Tratamiento da una solución objetiva y no mágica a la lesión, lo que diferencia a este papiro de muchos otros que resuelven los casos con hechicerías y conjuros. Finalmente, están las Glosas y Comentarios, que son principalmente la explicación de terminología que en el papiro se llama "antigua", lo que respalda la teoría de que el papiro original data de una fecha mucho más anterior.

\section{Trascendencia Médica y Odontológica}

Los aportes del Papiro de Edwin Smith a la medicina y odontología son variados y muy valiosos. En él se observa el nacimiento del quehacer científico en la medicina antigua. Se fundamenta en la observación, recolección y clasificación de los hechos y en la aplicación de un proceso mental inductivo, además de consistir en el primer tratado de cirugía conocido ${ }^{7}$.

En él se destaca el uso de la semiología para el estudio de las enfermedades, metodología emergente y poco practicada en esa época (Tabla 1). La sistemática utilizada en el papiro se asemeja bastante a la utilizada actualmente, tanto en lo referente a la anamnesis como al plan de tratamiento $\mathrm{y}$ al tratamiento propiamente tal.

En los párrafos del papiro fue donde primero se usaron las palabras cerebro, meninges, suturas craneanas y líquido céfalo raquídeo, constituyendo las bases de la primera nomenclatura anatómica.

El símil que es utilizado por el cirujano para describir las circunvoluciones y giros del cerebro es "el aspecto que ofrece una sustancia corrugada similar a la del cobre fundido". En varios casos expuestos, nota las pulsaciones y el aleteo que son 
Tabla 1. Esquema semiológico utilizado en el Papiro de Edwin Smith

1. Título: Se identifica la zona lesionada y órganos afectados

2. Examen:

a. Interrogatorio: La historia de los síntomas, las respuestas dadas por el paciente, las manifestaciones dolorosas

b. Inspección: Observa las lesiones, nota los cambios de color o de expresión de la cara, signos olfatorios

3. Palpación: Usa una fórmula introductoria: "Si tú examinases a un hombre que tenga...", "Tú deberías explorar su herida"

4. Ordena realizar pruebas especiales: levantar la cara, dirigir selectivamente la mirada, estirar las piernas

5. Diagnóstico: Utiliza formatos preestablecidos

6. Establece la conducta a seguir: De acuerdo con la gravedad del padecimiento. "El punto decisivo"

7. Tratamiento:

a. Exclusivamente mecánico o quirúrgico (3 casos)

b. Combinación de tratamiento quirúrgico con el uso externo de medicamentos (20 casos)

8. Uso exclusivo de medicamentos externos (19 casos)

9. Utiliza por primera vez el adhesivo y la sutura quirúrgica

percibidos por el dedo del cirujano. Aparentemente, es un signo que indica que no hay una situación de tensión intracraneana (Tabla 2).

Por primera vez, se destaca con tal importancia el sistema nervioso central y el cerebro como órgano rector de las funciones corporales. Se menciona la relación entre cerebro, médula espinal y las consecuencias producidas por daño a estas estructuras, atribuyéndose la parálisis de las extremidades inferiores a lesiones cerebrales, estableciéndose la relación entre la localización de la lesión craneal y el lado del cuerpo involucrado y observándose daños motores y sensoriales en las lesiones por compresión de las vértebras cervicales ${ }^{3}$. En las heridas donde se involucran las meninges o el cerebro se observa la irritación meníngea en forma de "anquilosamiento" del cuello o la incapacidad de los pacientes de "mirar sus hombros".

Por regla general, se describe el sangrado de los oídos y de las fosas nasales acompañando a las fracturas de cráneo y el modo de andar "arrastrando los pies” después de un daño cerebral.

\section{Tabla 2. Caso seis: Una herida abierta con fractura complicada, conminuta del cráneo y con ruptura de las membranas meníngeas}

\section{Título:}

Instrucciones relativas a una herida a su cabeza, la cual penetra hasta el hueso y deja expuesto al cerebro contenido en su cráneo

\section{Examen:}

Si tú examinases un hombre que presenta una herida abierta en su cabeza, la cual penetra hasta el hueso, en relación con un golpe recibido en su cráneo (el cual) expone al cerebro de su cráneo, tú deberás palpar su herida. Si tú encuentras que ese golpe dado a su cráneo, (expone como) esas arrugas (está corrugado) y adopta la forma del cobre fundido y percibes algo dentro que pulsa y aletea bajo tus dedos, como ocurre en el sitio débil de la corona del niño (la fontanela), la cual cuando se cierra ya no presenta esa pulsación ni ese aleteo sino hasta (cuando) el cráneo de su paciente se presenta abierto, y él sangra por sus fosas nasales y él sufre de rigidez de su cuello

\section{Diagnóstico:}

Tú deberás decir "es un padecimiento que no debe ser tratado"

\section{Tratamiento:}

Tú deberás untar la herida con grasa. Tú no deberás vendarla; tú no aplicaras dos cintas adhesivas: hasta que tú sepas que él ha alcanzado el punto decisivo

Estudia la función cardiovascular mediante la palpación del pulso y el recuento de las pulsaciones para evaluar la respuesta del organismo y se describen buenos conocimientos sobre función gástrica, vejiga, sistema cardiovascular y aspectos neuroquirúrgicos ${ }^{8}$.

Se relatan casos de heridas, fracturas, luxaciones, úlceras, tumores y abscesos y sus consecuencias, así como también describe por primera vez los métodos de reducción de fractura y luxación y los procedimientos para su contención. Cabe destacar dentro del papiro la dislocación de la mandíbula, cuya reducción se describe de manera idéntica a como se realiza actualmente, constituyendo un gran aporte a la historia del quehacer odontológico (Tabla 3).

Postula por primera vez el uso de adhesivo, sutura quirúrgica y cauterización como métodos de sinéresis. Y a diferencia de los remedios mágicos utilizados en la época, en el papiro se utiliza por primera vez el salicilato como analgésico y agente antiinflamatorio. 


\section{Tabla 3. Caso 25: Dislocación de la mandíbula}

Título:
Instrucciones con respecto a una dislocación de mandíbula

\section{Examen:}

Si usted examina un hombre con la mandíbula dislocada, debe ver si puede abrirle la boca y si también puede cerrársela. Debe colocar sus pulgares sobre los extremos de las dos ramas mandibulares dentro de la boca y sus dos pinzas (dos grupos de dedos) debajo de la barbilla y debe hacerlas retroceder hasta lograr que descansen en su lugar

\section{Diagnóstico:}

En relación con él usted debe decir que la dislocación de la mandíbula es una dolencia que trataré

\section{Tratamiento:}

Usted debe vendarlo con ymrn* y miel todos los días hasta lograr su recuperación

* Se desconoce la naturaleza del medicamento aplicado ymrw habiéndose especulado que podría tratarse de un medicamento mineral que podría actuar como desinfectante ${ }^{7}$.

La carne fresca es el vendaje usual para el primer día, quizás por su valor hemostático. Después se aplican los calmantes y las sustancias protectoras y tanto el calor como el frío se utilizan en condiciones indicadas por la lógica ${ }^{8}$. El uso de apósitos médicos se observa por primera vez en este documento. En veintidós casos del papiro se utilizó una combinación de grasa con miel a modo de ungüento y en 17 de estos casos también se utilizó gasa. Se cree que este vendaje podría tratar de evitar la contaminación producida por las moscas?.

En el papiro también se comienza a trabajar el concepto que ahora conocemos como plan de tratamiento, en el sentido de que ante un determinado problema, en el papiro se plantean tres diferentes opciones para tratar al enfermo, eligiendo aquella que proporcione mejores opciones de recuperación y en relación a los materiales disponibles: La primera alternativa se refiere al tratamiento exclusivamente mecánico o quirúrgico (tres casos descritos en el papiro), para los cuales se describe el uso de torundas, hisopos de lino, vendas de lino, algún tipo de tela adhesiva, suturas quirúrgicas y cauterización (descritas por primera vez en la literatura médica) y entablillados, muletas y soportes para mantener al paciente en posición erecta. La segunda opción de tratamiento es la combinación del quirúrgico con el uso externo de medicamentos ( 20 casos). Y la tercera opción es la utilización de medicamentos externos (19 casos).

Como una parte final del tratamiento $-y$ no en todos los casos-, se menciona de manera sistemática una serie de cláusulas de temporalidad, que se relacionan directamente con la condición del enfermo, y son tres: a) hasta que se recupere; b) hasta que el período de su enfermedad pase; $y$, c) hasta que alcance un punto decisivo.

\section{La relevancia del papiro de Edwin Smith}

La pregunta es, ¿habrá tenido alguna influencia la medicina egipcia en el posterior desarrollo de la medicina grecorromana?

Ampliamente difundida se encuentra la idea de que las bases de la medicina tal como la conocemos hoy en día fueron establecidas durante el período grecorromano. Esto se ve apoyado en la gran cantidad de registros que se tienen de estas civilizaciones.

No se ha considerado demasiado el desarrollo de la medicina egipcia como un avance importante, puesto que regularmente se ha pensado que esta tenía un origen netamente mágico-religioso.

Los conocimientos de los egipcios acerca de la anatomía humana no se comparan con el posterior desarrollo que se alcanzó en esta disciplina. Esto debido probablemente al valor sacro que se le atribuía a los muertos en esta época y al poco contacto que se mantuvo por mucho tiempo con otras culturas.

Se ha pensado que la gran diferencia que había entre los conocimientos que se tenían en el antiguo Egipto y el posterior avance que presentó la medicina grecorromana hace la gran diferencia entre ellas, $y$ es por esto que la medicina egipcia no se puede considerar relevante. Sin embargo, es importante notar que la mayor parte de los documentos de esta época se perdieron o bien fueron quemados junto con la gran biblioteca de Alejandría. En adición a esto, sabemos que los conocimientos han cambiado durante la historia y siguen cambiando aceleradamente en el campo de la salud.

El hecho que marca el comienzo de la práctica médica es un cambio en el método. El estudio de la medicina en el antiguo Egipto (como se muestra en el papiro de Smith) se vuelve riguroso y se independiza de los fenómenos sobrenaturales. 
Los hallazgos médicos se tornan el resultado de una observación sistemática, el conocimiento se vuelve empírico.

El inicio en la medicina como hoy la conocemos no comienza por la acumulación suficiente de conocimientos, sino con la incorporación del método científico a su estudio.

\section{Conclusión}

La importancia del papiro de Smith es por una parte la de ilustrarnos acerca de cómo pudo haberse practicado la medicina en el antiguo Egipto, pero lo más relevante es que constituye el primer documento de carácter médico, en la historia, que refleja un estudio basado en la observación objetiva, en el que se excluyen todas las percepciones mágico-religiosas, así como el marco cultural propio.

La teoría de que los conocimientos de la medicina egipcia fueron traspasados a Grecia, y pudieron ser importantes para el desarrollo de la posterior medicina hipocrática, se hace más fuerte cuando encontramos referencias a la medicina egipcia tanto en $\mathrm{La}$ Odisea de Homero (s. VIII a.C.), así como en la historia de Heródoto (484-425 a.C.) que incluso describe las distintas especialidades que existían ${ }^{10}$.

La semejanza que existe entre el actual método clínico y lo descrito en el papiro de Smith, hace pensar fuertemente en la idea de que el origen de la medicina, o al menos un esbozo de ella, pudo haberse encontrado en el antiguo Egipto

\section{Referencias}

1. Porter R. The Greatest Benefit to Mankind: a medical history of humanity. New York: Editorial Norton \& Company; 1998. pp. 831.

2. Ghalioungui P. La medicina del Egipto faraónico. En: Lain Entralgo, P. Editor, Historia Universal de la Medicina. Barcelona, España: Salvat Editores; 1972. p. 124.

3. Stiefel M, Shaner A, Shaefer S. The Edwin Smith Papyrus: the birth of analytical thinking in medicine and Otolaryngology. Laryngoscope 2006; 116 (2): 182-8.

4. López Espinosa JA. Una rareza bibliográfica universal: el Papiro médico de Edwin Smith. ACIMED 2002; 10 (3): 9-10. Disponible en www.scielo.sld.cu [Consultado el 12 de octubre de 2011].

5. González Fisher R, Flores P. El Papiro Quirúrgico de Edwin Smith. An Med Asoc Med Hosp 2005; 50 (1): 43-8.

6. The Edwin Smith Papyrus. Versión digital en Turning the Pages. Disponible en http://archive.nlm.nih.gov [Consultado el 13 de noviembre de 2011].

7. Puigbo JJ. El papiro de Edwin Smith y la civilización egipcia. Gac Méd Caracas 2002; 110 (3): 378-85.

8. Krivoy A, Krivoy J, Krivoy M. Aspectos neuroquirúrgicos parciales del Papiro de Edwin Smith. Gac Med Caracas 2002; 110 (3): 386-91.

9. Puigbo JJ. El Papiro de Edwin Smith "Una obra maestra de la medicina del antiguo Egipto". Traducción del libro del Prof. J. Breasted. Gac Med Caracas 2002; 110 (2): 253-75.

10. Nunn JF. La Medicina del Antiguo Egipto. México: Editorial Fondo de Cultura Económico; México. pp 288. 